\title{
Online Influence Maximization
}

Siyu Lei, Silviu Maniu, Luyi Mo, Reynold Cheng, Pierre Senellart
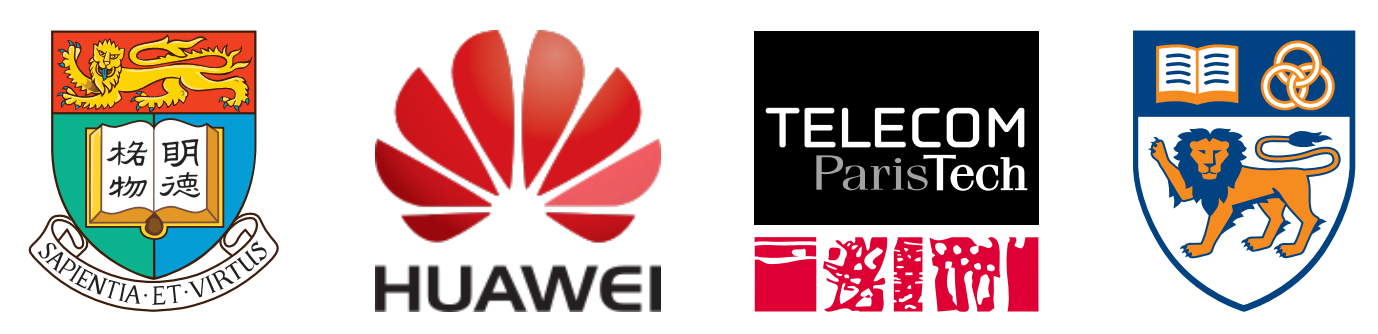

KDD 2015, Sydney, Australia August 13, 2015 


\section{Influence Maximization}

Important problem in social networks, with applications in marketing, computational advertising

- objective: given a promotion budget, maximize the influence spread in the social network (word-ofmouth effect)

select $k$ seeds (influencers) in the social graph, given an influence graph and a propagation model 


\section{Influence Maximization}

Data model: influence graph $G(V, E, p)$, where

- $V$ and $E$ and the vertices (users) and edges (follow relations, friendship, etc.) in the social network,

- $p$ is a function mapping edges to influence probabilities. 


\section{Influence Maximization}

Independent cascade model - a discrete time model of propagation:

- at time $\mathrm{O}$ - activate the seed $\mathrm{s}$,

- node $i$ activated at time $t$ - influence is propagated at $t+1$ to neighbours $j$ independently with probability $p(i, j)$,

- once a node is activated, it cannot be deactivated or activated again. 


\section{Influence Maximization}

The independent cascade model is a stochastic process

Influence maximization in this model tries to optimize the expected influence spread, $\sigma(S)$, from a set of seeds $S$. 


\section{Influence Maximization}

Influence maximization is computationally hard - two sources of hardness:

- computing $\sigma(S)$ is hard = evaluating probability formulas

- even if we know $\sigma(S)$, computing the influence maximisation is NPhard (submodular maximization subject to a constraint)

Solutions:

- for computing $\sigma(S)$ : Monte Carlo simulations of influence spread

- for solving the influence maximization: greedy approximation algorithm

Multiple algorithms and estimators: CELF, TIM / TIM+ 


\section{Online Influence Maximization (OIM)}

What if we only know the social graph, but still want to maximize influence, with a budget?

- we need to keep an (uncertain) model of the influence graph

- classic trade-off between exploration (refine the model) and exploitation (use the model to maximize influence)

- lends itself to an iterative process over several rounds (online) 


\section{Online Influence Maximization Problem}

Maximize the influence spread given a budget of $N$ rounds of choosing $k$ seeds in the network

- Contribution: an online framework - maximization and model refinement over multiple rounds 


\section{OIM Framework}

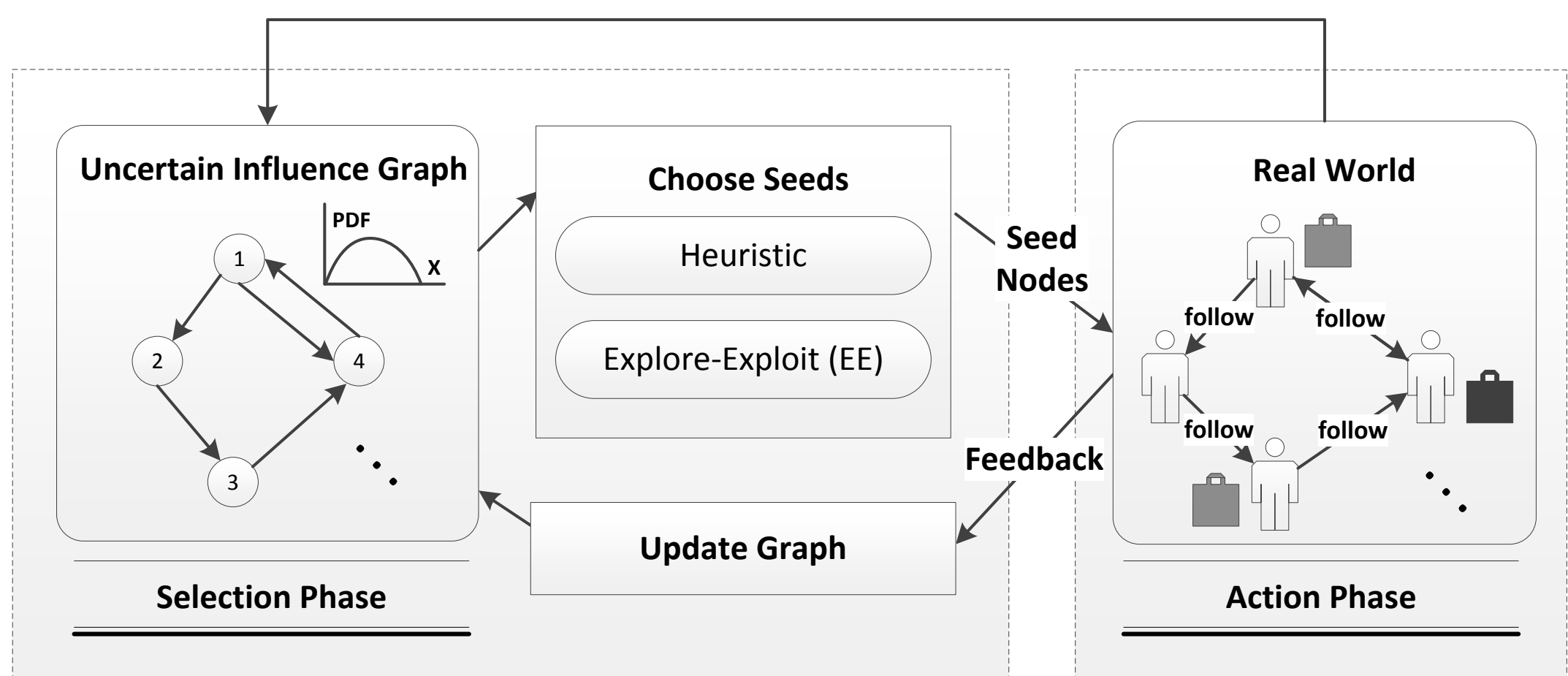

1: Input: \# trials $N$, budget $k$, uncertain influence graph $G$

2: Output: seed nodes $S_{n}(n=1 \ldots N)$, activation results $A$

3: $A \leftarrow \emptyset$

4: for $n=1$ to $N$ do

5: $\quad S_{n} \leftarrow$ Choose $(G, k)$

6: $\quad\left(A_{n}, F_{n}\right) \leftarrow$ RealWorld $\left(S_{n}\right)$

7: $\quad A \leftarrow A \cup A_{n}$

8: $\quad \operatorname{Update}\left(G, F_{n}\right)$

9: return $\left\{S_{n} \mid n=1 \ldots N\right\}, A$ 


\section{OIM Framework}

Three ingredients:

- the model of the influence graph

- the explore-exploit strategy (Choose)

- after real-world feedback, update of the model (Update) 


\section{Uncertain Influence Graph}

Probabilistic graph model:

- instead of a probability $p(i, j)$ on each edge $(i, j)$, we associate it with a distribution of probabilities

$$
P(i, j) \sim \operatorname{Beta}\left(\alpha_{i j}, \beta_{i j}\right)
$$

- by default, each edge is associated with a prior probability distribution $\operatorname{Beta}(\alpha, \beta)$ 


\section{Choose Strategies}

The uncertain graph model allows us to explore different assumptions about the graph:

- exploit assumes that the influence probabilities are the expected value of $P(i, j)$

- explore uses either other assumptions about the graph, or uses heuristic strategies (random, max degree, degree discount)

For each branch, the IM algorithm is a black box (CELF, $\mathrm{TIM}, . .$.$) only the input influence graph is different$ 


\section{Choose: Confidence Bound}

A classic approach to use other assumptions about the influence graph is the Confidence Bound (CB) algorithm:

- each edge distribution is "moved" by $\theta$

1: Input: uncertain influence graph $G=(V, E, P)$, budget $k$

2: Output: seed nodes $S$ with $|S|=k$ standard deviations, and the IM algorithm is $\begin{aligned} & 3: \text { for } e \in E \mathbf{d o}_{\alpha_{i j}} \\ & 4: \quad \mu_{i j} \leftarrow \frac{\alpha_{i j}}{\alpha_{i j}+\beta_{i j}}\end{aligned}$ executed

5: $\quad \sigma_{i j} \leftarrow \frac{1}{\left(\alpha_{i j}+\beta_{i j}\right)} \cdot \sqrt{\frac{\alpha_{i j} \beta_{i j}}{\left(\alpha_{i j}+\beta_{i j}+1\right)}}$

6: $\quad p_{i j} \leftarrow \mu_{i j}+\theta \sigma_{i j}$

- allows to "explore" other "possible influence 7: $G^{\prime} \leftarrow G$, with edge probabilities $p_{i j}, \forall(i, j) \in E$ graphs"

8: $S \leftarrow \operatorname{IM}\left(G^{\prime}, k\right)$

9: return $S$

- exploit corresponds to the case where $\theta$ is 0

A probabilistic parameter $\varepsilon$ allows the choice between different $\theta$ values (including 0 for exploit) — similar to $\varepsilon$-greedy 


\section{Choose: Confidence Bound}

Advantages of CB:

- allows the update of $\varepsilon$ probabilities for a fixed choice of $\theta$ values Exponentiated Gradient (EG)

- using CB with EG allows a theoretical regret bound for a given choice of (constant) $\theta$ values
1: Input: $\vec{\varphi}$, probability distribution; $\delta$, accuracy parameter; $G_{n}$, the gain obtained; $j$, the index of latest used $\theta_{j}$; w, a vector of weights; $N$, the number of trials.

2: Output: $\theta$

3: $\gamma \leftarrow \sqrt{\frac{\ln (q / \delta)}{q N}}, \tau \leftarrow \frac{4 q \gamma}{3+\gamma}, \lambda \leftarrow \frac{\tau}{2 q}$

4: for $i=1$ to $q$ do

5: $\quad w_{i} \leftarrow w_{i} \times \exp \left(\lambda \times \frac{G_{n} \times \mathbb{I}[i=j]+\gamma}{\varphi_{i}}\right)$

6: for $i=1$ to $q$ do

7: $\quad \varphi_{i} \leftarrow(1-\tau) \times \frac{w_{i}}{\sum_{j=1}^{k} w_{j}}+\tau \times \frac{1}{q}$

8: return sample from $\vec{\theta}$ according to $\vec{\varphi}$ distribution 


\section{Real-World Feedback}

Once a strategy has been chosen and a seed set identified:

- we test $\mathrm{S}$ in the real-world (posting on Twitter, flyers in a city,...)

- in round $\mathrm{n}$, we get activation feedback composed of activated nodes $A_{n}$, and feedback set $F_{n}$ tuples $\left(i, j, a_{i j}\right)$ for every affected edge 


\section{Update Step}

Two approaches to Update:

- local update: each edge in the feedback is updated in a Bayesian manner

- global update: each edge in the graph is updated using methods such as maximum likelihood or least squares regression

Feedback $A_{n}, F_{n}:\left(i, j, a_{i j}\right)$

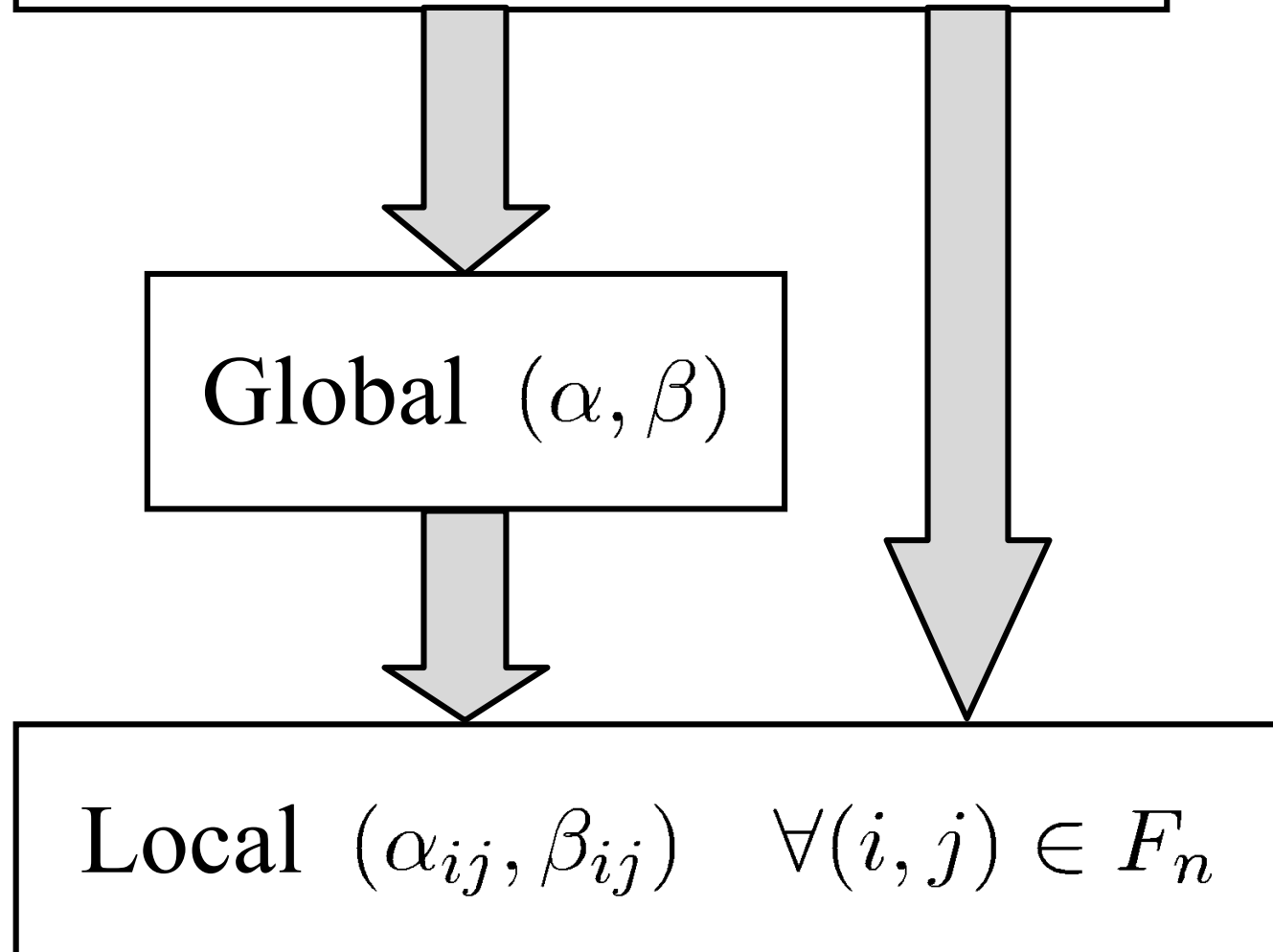

- can also be combined 


\section{Local Update}

Beta distribution is a conjugate prior of the Bernoulli distribution — the update is straightforward:

- success $a_{i j}=1 \Longrightarrow P_{i j} \sim \operatorname{Beta}\left(\alpha_{i j}+1, \beta_{i j}\right)$

- failure $a_{i j}=0 \Longrightarrow P_{i j} \sim \operatorname{Beta}\left(\alpha_{i j}, \beta_{i j}+1\right)$

- same as counting the number of successful and failed activations for each edge 


\section{Global Update}

Only using local update might be too sparse especially for low influence probabilities, can lead to over reliance on the prior.

Solution: update also the prior for all edges, using all the feedback history 


\section{Global Update}

Ordinary Least Squares (LSE): update via least squares estimation, from the formula of a spread of a node:

$$
\sigma_{n}(\{s\})=1+\sum_{\substack{(s, i) \in E \\ i \notin \mathcal{A}_{n}}} p_{s i} \times \sigma_{n}(\{i\})+\sum_{\substack{(s, i) \in E \\ i \in \mathcal{A}_{n}}} p_{s i} \times\left(\sigma_{n}(\{i\})-1\right)
$$

which leads to

$$
\begin{gathered}
\left(\left|A_{n}\right|-1\right) \beta=\left(1-\left|A_{n}\right|\right)\left(t_{s}+1\right)+\left(h_{s}+o_{s}\right) \hat{\sigma}_{n}-\left(h_{a s}+a_{s}\right) \\
x_{n} \beta=y_{n} . \\
\hat{\beta}=(\vec{x} \cdot \vec{y}) /(\vec{x} \cdot \vec{x})
\end{gathered}
$$




\section{Global Update}

Maximum Likelihood (MLE): assume edges are independent:

$$
\mathcal{L}\left(F_{n} \mid \alpha, \beta\right)=\prod_{\left(i, j, a_{i j}\right) \in F_{n}} \frac{\left(\alpha+h_{i j}\right)^{a_{i j}}\left(\beta+m_{i j}\right)^{1-a_{i j}}}{\alpha+\beta+h_{i j}+m_{i j}} .
$$

and the parameters can be estimated from

$$
\sum_{\left(i, j, a_{i j}\right) \in F_{n}, a_{i j}=1} \frac{1}{\alpha+h_{i j}}=\sum_{\left(i, j, a_{i j}\right) \in F_{n}, a_{i j}=0} \frac{1}{\beta+m_{i j}}
$$




\section{Sampling Optimization}

Even advanced algorithms rely on sampling for influence estimation — costly over multiple rounds

- incremental optimization approach - reuse of samples between rounds in little-affected parts of the graph

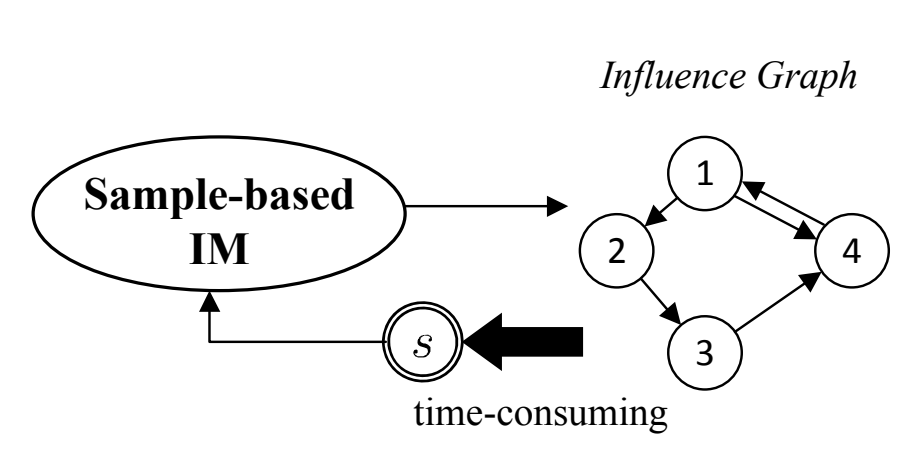

(a) Non-incremental

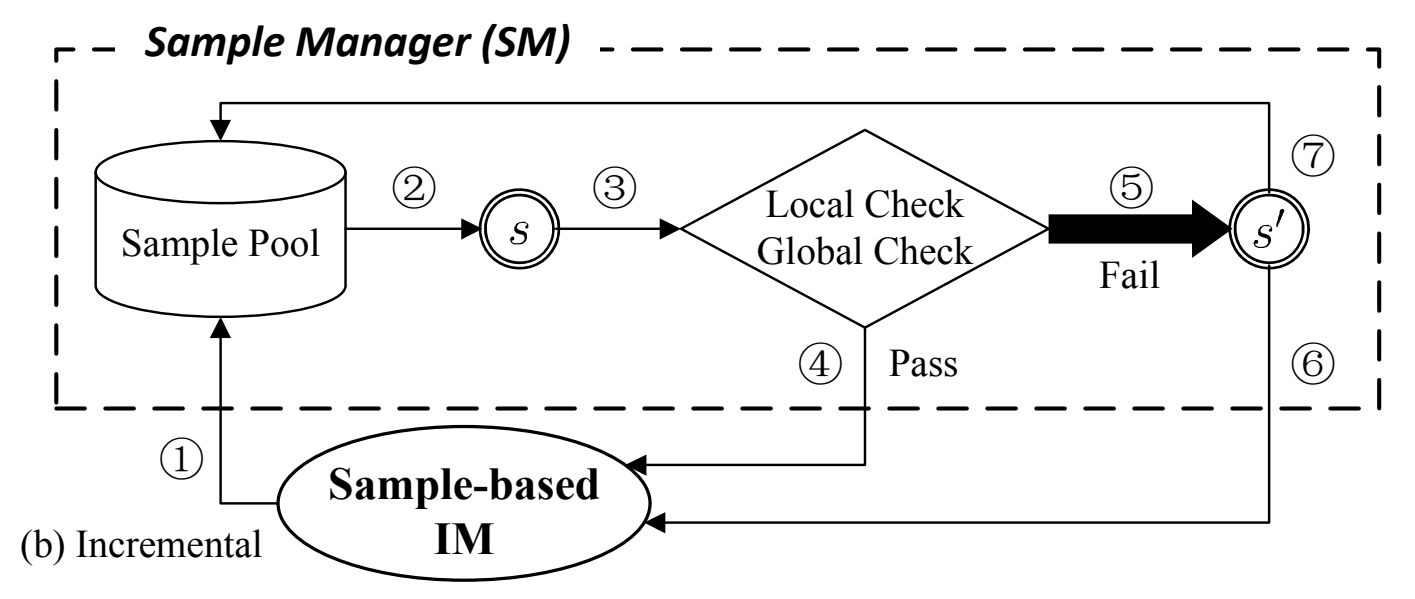

sample pool

(s): $\left(\alpha^{t}, \beta^{t}\right), \operatorname{trial}($ or age $) t$

node activated history

(14) : latest activated trial(or age) $l$

(c) Data in SM 


\section{Results: effectiveness of explore-exploit strategies}
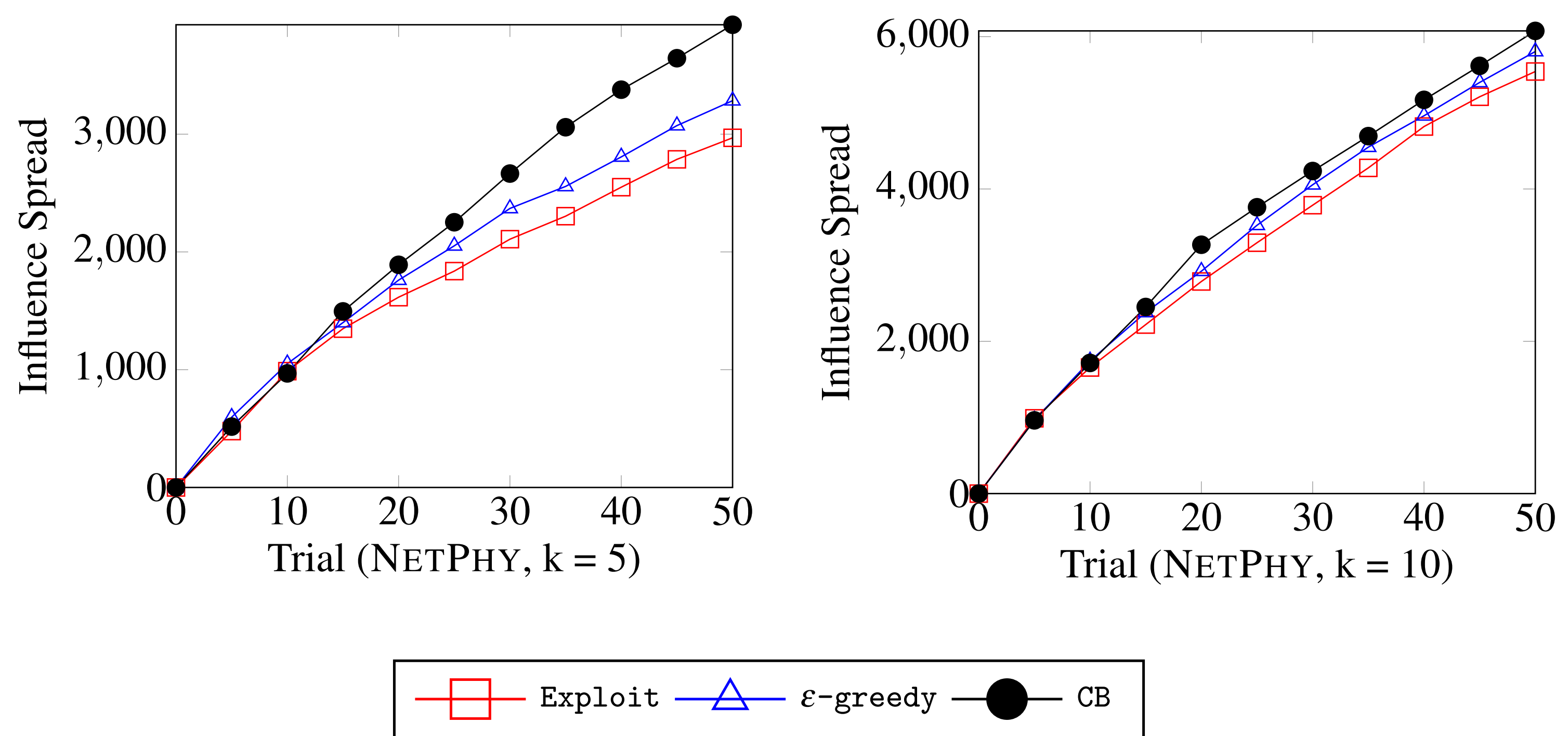


\section{Results: effectiveness of update methods}

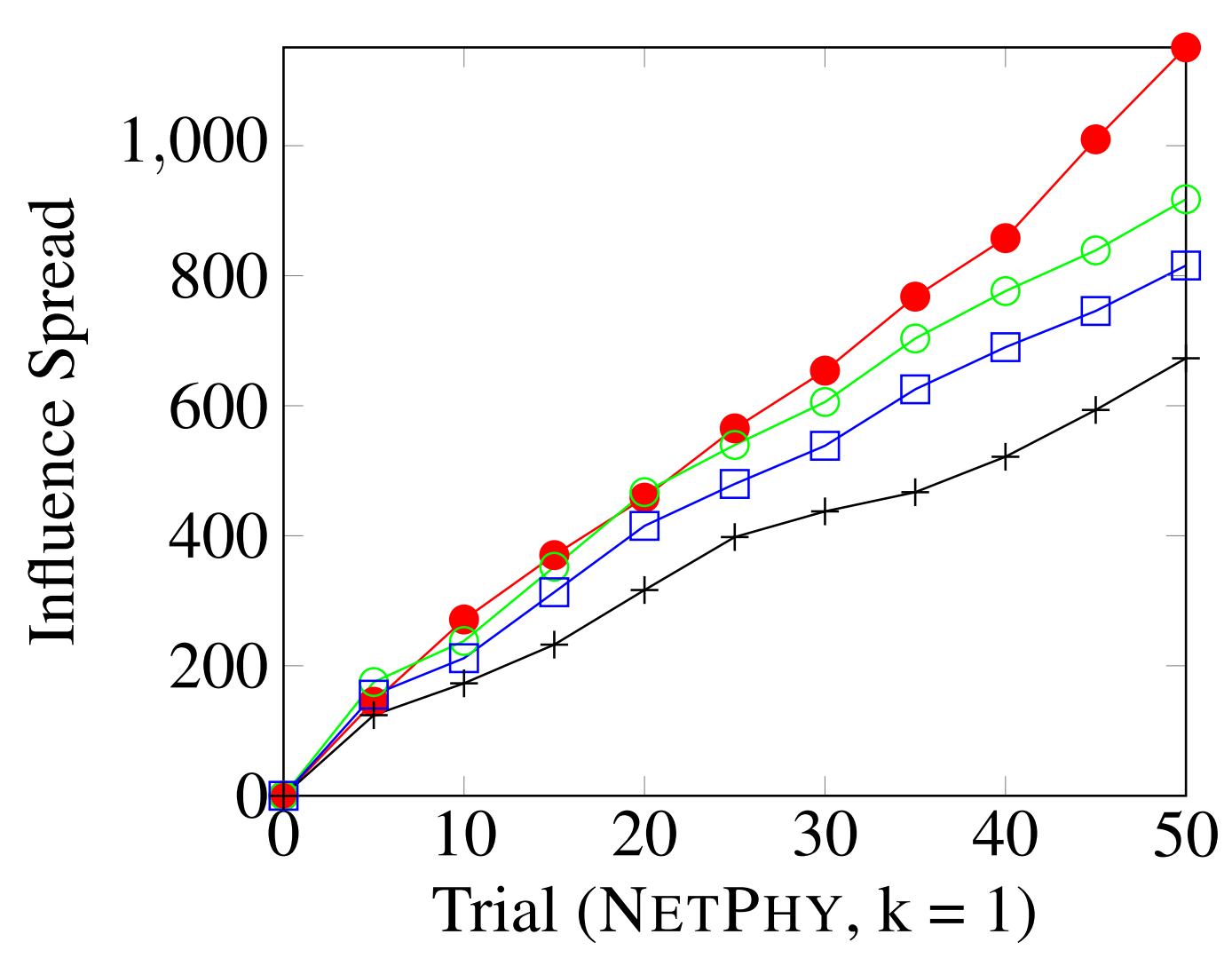

(a) Different updates

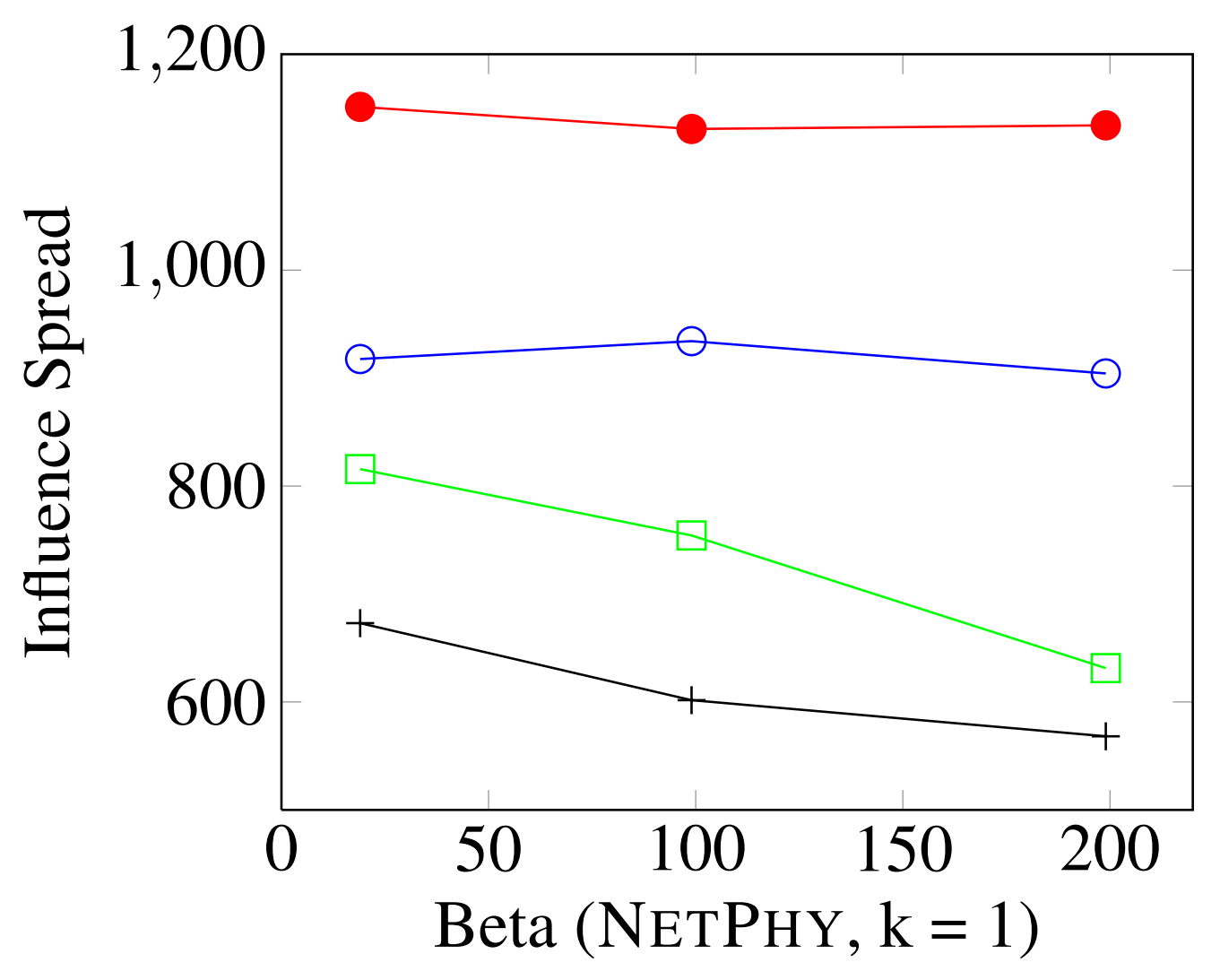

(b) Effect of priors

$$
-{ }_{\mathrm{CB}+\mathrm{MLE}}-\mathrm{O} \text { CB+LSE } \square{ }^{\mathrm{CB}+\mathrm{LOC}}-\mathrm{CB}^{\mathrm{CB}}+\mathrm{NO}
$$




\section{Results: effectiveness versus heuristics}
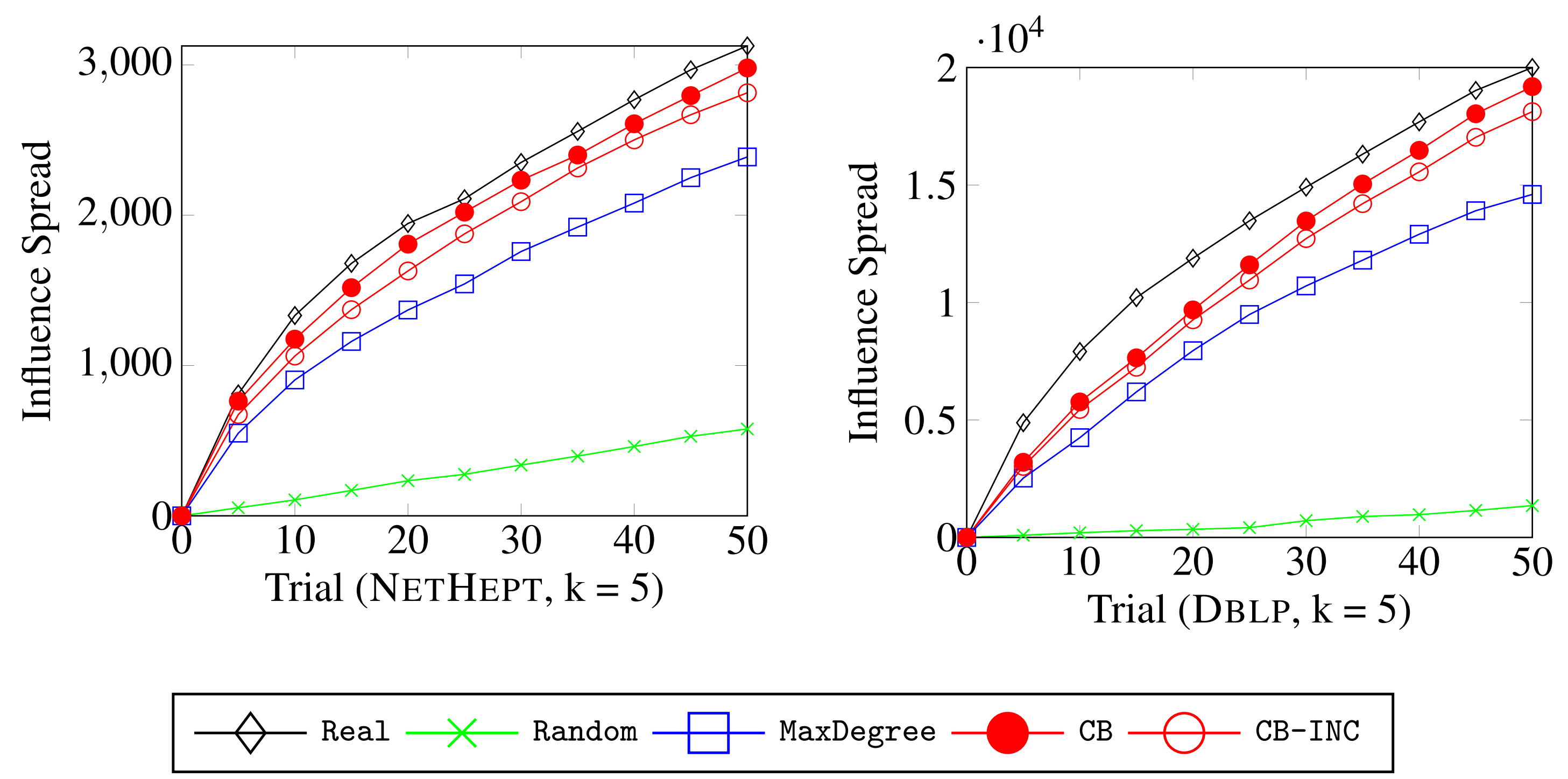


\section{Results: efficiency of sample reuse}
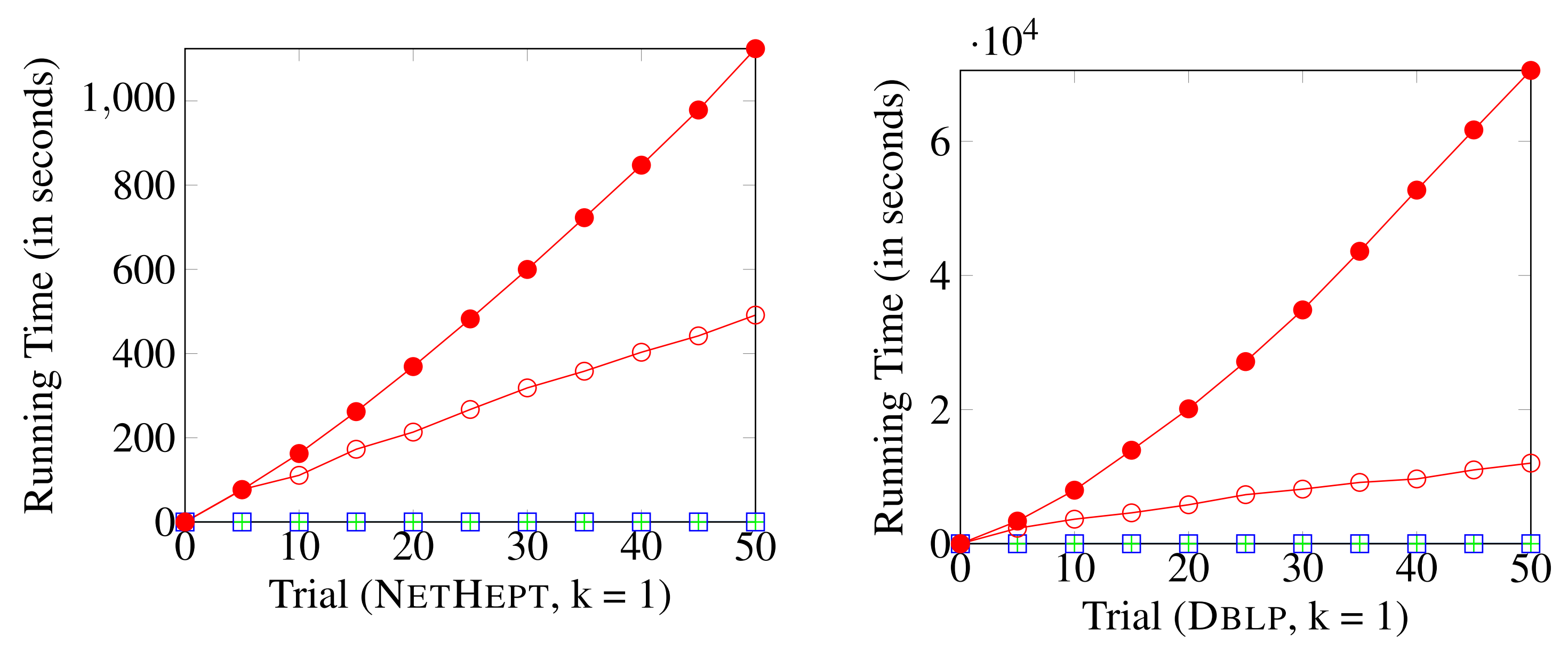

- Random $\square$ MaxDegree $-\mathrm{O}$ CB $\bigcirc$ CB-INC 


\section{Research Perspectives}

- scalability is still a big issue in influence maximisation - even more so in the online setting

- adapting the framework to other influence models (threshold, credit distribution)

- learning also the influence model - do not rely on "synthetic" models such as independent cascade and threshold 